Magnolia Petroleum Company

See SM (Socony Mobil Oil Company), page 145.

\title{
Riken (Tokyo), the Institute of Physical and Chemical Research
}

$\begin{array}{ccc}\begin{array}{c}\text { Laboratory } \\ \text { number }\end{array} & \begin{array}{c}\text { Published } \\ \text { reference }\end{array} & \begin{array}{c}\text { Original date } \\ \text { or other value }\end{array} \\ \mathrm{N}-52 & 129: 113 & 1490 \pm 160 \\ -53 & 129: 114 & 2700 \pm 170 \\ -57 & 129: 114 & 1220 \pm 130 \\ -59 & 129: 114 & 3780 \pm 150 \\ -61 & 129: 114 & 1430 \pm 150 \\ -68 & 129: 114 & 1890 \pm 120 \\ -70 & 129: 113 & 1950 \pm 130 \\ -71 & 129: 113 & 1940 \pm 120 \\ -73 & 129: 113 & 1940 \pm 100 \\ -76 & 129: 113 & 2280 \pm 120 \\ -77 & 129: 113 & 1990 \pm 120 \\ -79 & 129: 113 & 2110 \pm 120 \\ -81-2 & 129: 113 & 1970 \pm 120 \\ -91 & 129: 114 & 178 \pm 100 \\ -93 & 129: 112 & 17,710 \pm 750 \\ -94 & 129: 115 & 3000 \pm 120 \\ -95 & 129: 112 & >36,800 \\ -96-1 & 129: 112 & >36,800 \\ -96-2 & 129: 112 & 21,470 \pm 1130 \\ -97-1 & 129: 112 & 22,720 \pm 800 \\ -97-2 & 129: 113 & 22,970 \pm 800\end{array}$

Corrected date
or other value

\section{National Physical Laboratory}

$\begin{array}{ccc}\begin{array}{c}\text { Laboratory } \\ \text { number }\end{array} & \begin{array}{c}\text { Published } \\ \text { reference }\end{array} & \begin{array}{c}\text { Original date } \\ \text { or other value }\end{array} \\ \text { NPL-10 } & 104: 35 & 3355 \pm 155 \\ -11 & 104: 35 & 3470 \pm 145 \\ -12 & 104: 35 & 3080 \pm 145 \\ -13 & 104: 35 & 4420 \pm 102 \\ -14 & 104: 35 & 4220 \pm 105 \\ -15 B & 122: 27 & 6500 \pm 108 \\ -16 & 122: 25 & 10,780 \pm 162 \\ -17 & 104: 36 & 3345 \pm 160 \\ -18 & 104: 36 & 3355 \pm 160 \\ -19 & 104: 36 & 3130 \pm 180 \\ -21 & 104: 36 & 3070 \pm 103 \\ -23 & 122: 26 & 3020 \pm 94 \\ -24 & 122: 26 & 3340 \pm 92 \\ -25 & 122: 26 & 1550 \pm 120 \\ -26 & 104: 36 & 3290 \pm 95 \\ -27 \mathrm{c} & 104: 36 & 10,530 \pm 156 \\ -28 & 122: 27 & 2950 \pm 91 \\ -29 & 122: 27 & 3170 \pm 94 \\ -30 & 104: 38 & 3400 \pm 97 \\ -31 & 104: 38 & 3600 \pm 93 \\ -32 & 104: 38 & 2550 \pm 90 \\ -33 & 104: 38 & 12,900 \pm 170 \\ -34 & 122: 26 & >43,500 \\ -35 & 122: 28 & 14,760 \pm 202 \\ -36 & 122: 28 & 10,720 \pm 132 \\ -37 & 122: 28 & 18,190 \pm 306 \\ -38 & 122: 28 & 31,480 \pm 1640 \\ -39 & 104: 37 & 4830 \pm 100 \\ -40 & 104: 37 & 5800 \pm 108\end{array}$

Corrected date
or other value

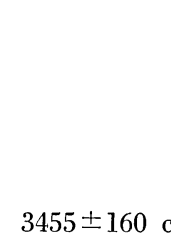

$3455 \pm 160 \mathrm{c}$

$$
\begin{array}{r}
\partial \mathrm{C}^{14}, \% \circ \\
-168.8 \pm 19.0 \\
-285.6 \pm 19.9 \\
-140.2 \pm 15.0 \\
-375.1 \pm 17.5 \\
-170.6 \pm 18.0 \\
-209.3 \pm 14.5 \\
-215.9 \pm 15.2 \\
-215.0 \pm 14.5 \\
-215.2 \pm 12.5 \\
-248.4 \pm 14.7 \\
-218.9 \pm 14.1 \\
-230.3 \pm 14.5 \\
-217.6 \pm 14.7 \\
-22.2 \pm 12.0 \\
-890.0 \pm 89.7 \\
-318.6 \pm 17.3 \\
-998.3 \pm 541.0 \\
-998.3 \pm 541.0 \\
-930.6 \pm 135.5 \\
-941.0 \pm 96.9 \\
-942.7 \pm 91.8
\end{array}
$$

A.D./ B.C. date A.D. 460 750 B.C. A.D. 730 1830 B.C. A.D. 520

A.D. 60

A.D. 0

A.D. 10

A.D. 10 330 B.C. 40 B.C.

160 B.C.

20 B.C.

A.D. 1772

15,760 B.C. 1050 B.C.

19,520 B.C 20,770 B.C. 21,020 B.C.

A.D./ B.C. date 1405 B.C. 1520 B.C. 1130 B.C. 2470 B.C. 2270 B.C. 4550 B.C. 8830 B.C. 1395 B.C. 1505 B.C. 1180 B.C. 1120 B.C. 1070 B.C. 1390 B.C. A.D. 400 1340 B.C. 8580 B.C. 1000 B.C. 1220 B.C. 1450 B.C. 1650 B.C. 600 B.C. 10,950 B.C.

12,810 B.C. 8770 B.C. 16,240 B.C. 29,530 B.C. 2880 B.C. 3850 B.C. 


\begin{tabular}{|c|c|c|c|c|c|}
\hline $\begin{array}{l}\text { Laboratory } \\
\text { number }\end{array}$ & $\begin{array}{l}\text { Published } \\
\text { reference }\end{array}$ & $\begin{array}{l}\text { Original date or } \\
\text { other value }\end{array}$ & $\begin{array}{l}\text { Corrected date } \\
\text { or other value }\end{array}$ & $\partial \mathrm{C}^{14}, \% 0$ & $\begin{array}{c}\text { A.D./ } \\
\text { B.C. date }\end{array}$ \\
\hline-41 & $104: 37$ & $4860 \pm 97$ & & $-452 \pm 2.9$ & 2910 B.C. \\
\hline-42 & $104: 37$ & $6370 \pm 103$ & & $-550 \pm 2.8$ & 4420 B.C. \\
\hline-43 & $104: 37$ & $12,750 \pm 173$ & & $-795 \pm 2.9$ & 10,800 B.C. \\
\hline-44 & $104: 37$ & $12,580 \pm 172$ & & $-791 \pm 3.0$ & 10,630 B.C. \\
\hline-47 & $122: 29$ & $10,770 \pm 156$ & & $-738 \pm 3.6$ & 8820 B.C. \\
\hline-48 & $122: 29$ & $11,310 \pm 168$ & & $-756 \pm 3.7$ & 9360 B.C. \\
\hline-49 & $122: 29$ & $39,160+6090$ & & $-992 \pm 2.9$ & 37,210 B.C. \\
\hline-50 & $\begin{array}{l}122: 29 \\
129\end{array}$ & $\begin{array}{l}>36,900 \\
6570+05\end{array}$ & & $(-989 \pm 3.6)$ & \\
\hline-52 & $122: 28$ & $6570 \pm 95$ & & $-559 \pm 2.6$ & 4620 B.C. \\
\hline-53 & $122: 28$ & $9340 \pm 100$ & & $-688 \pm 2.0$ & 7390 B.C. \\
\hline-54 & $122: 28$ & $12,890 \pm 130$ & & $-710 \pm 2.5$ & 10,940 B.C. \\
\hline-55 & $122: 26$ & $32,160+1780$ & & $-982 \pm 2.3$ & 30,210 B.C. \\
\hline .56 & $122: 27$ & $10,560 \pm 142$ & & $-733 \pm 3.0$ & 8610 B.C. \\
\hline-57 & $122: 27$ & $3440 \pm 90$ & & $-350 \pm 2.4$ & 1490 B.C. \\
\hline .58 & $159: 160$ & $6900 \pm 130$ & & $-599 \pm 2.7$ & 4950 B.C. \\
\hline .59 & $159: 160$ & $525 \pm 100$ & & $-73 \pm 6.0$ & A.D. 1425 \\
\hline-60 & $122: 29$ & $700 \pm 85$ & & $-91 \pm 1.6$ & A.D. 1250 \\
\hline-61 & 159:161 & $1190 \pm 90$ & & $-140 \pm 2.4$ & A.D. 760 \\
\hline-62 & $159: 157$ & $410 \pm 90$ & & $-58 \pm 3.7$ & A.D. 1540 \\
\hline-63 & 159:159 & $3450 \pm 90$ & & $-353.9 \pm 2.2$ & 1500 B.C. \\
\hline-64 & 159:159 & $9410 \pm 100$ & & $-692.4 \pm 2.2$ & 7460 B.C. \\
\hline-65 & $159: 159$ & $3830 \pm 90$ & & $-379.7 \pm 2.5$ & 1880 B.C. \\
\hline-66 & 159:159 & $5020 \pm 90$ & & $-465.1 \pm 2.5$ & 3070 B.C. \\
\hline-67 & 159:159 & $12,610 \pm 110$ & & $-791.7 \pm 1.8$ & 10,660 B.C. \\
\hline-68 & $159: 160$ & $16,130 \pm 140$ & & $-865.8 \pm 1.9$ & 14,180 B.C. \\
\hline-69 & $159: 157$ & $3760 \pm 90$ & & $-375 \pm 2.5$ & 1810 B.C. \\
\hline-70 & $159: 161$ & $1810 \pm 95$ & & $-203 \pm 3.8$ & A.D. 140 \\
\hline-71 & $159: 160$ & $3900 \pm 125$ & & $-418 \pm 2.5$ & 1950 B.C. \\
\hline .73 & $159: 157$ & $5030 \pm 90$ & & $-465 \pm 1.9$ & 3080 B.C. \\
\hline-74 & $159: 158$ & $3330 \pm 90$ & & $-343 \pm 2.5$ & 1380 B.C. \\
\hline .75 & $159: 158$ & $3590 \pm 90$ & & $-361 \pm 2.8$ & 1640 B.C. \\
\hline-76 & $159: 158$ & $4740 \pm 90$ & & $-446 \pm 2.5$ & 2790 B.C. \\
\hline .77 & $159: 158$ & $3960 \pm 110$ & & $-389 \pm 5.7$ & 2010 B.C. \\
\hline-78 & $159: 158$ & $28,140+480$ & & $-970 \pm 1.7$ & 26,190 B.C. \\
\hline .79 & $159: 160$ & $39,050+1450$ & & $-992 \pm 1.2$ & 37,100 B.C. \\
\hline-80 & $159: 158$ & $\begin{array}{r}37,960+1700 \\
-1400\end{array}$ & & $-991 \pm 1.7$ & 36,010 B.C. \\
\hline-81 & $159: 159$ & $11,790 \pm 140$ & & $-770 \pm 3.3$ & 9840 B.C. \\
\hline
\end{tabular}

\section{New South Wales, Department of Nuclear and Radiation Chemistry}

\begin{tabular}{|c|c|c|c|c|c|}
\hline $\begin{array}{c}\text { Laboratory } \\
\text { number }\end{array}$ & $\begin{array}{l}\text { Published } \\
\text { reference }\end{array}$ & $\begin{array}{l}\text { Original date or } \\
\text { other value }\end{array}$ & $\begin{array}{l}\text { Corrected date } \\
\text { or other value }\end{array}$ & $\partial \mathrm{C}^{14}, \%$ & $\begin{array}{l}\text { A.D./ } \\
\text { B.C. date }\end{array}$ \\
\hline NSW-1 & $160: 163$ & $285 \pm 80$ & & & A.D. 1665 \\
\hline-2 & $160: 163$ & Modern & & & \\
\hline-3 & $160: 163$ & $>35,000$ & & & \\
\hline-4 & $160: 163$ & Modern & & & \\
\hline-5 & $160: 163$ & $550 \pm 85$ & & & A.D. 1400 \\
\hline-6 & $160: 163$ & $1220 \pm 55$ & & & A.D. 730 \\
\hline-8 & $160: 164$ & $3.31 \pm 0.40 \%$ & modern & & \\
\hline-9 & $160: 164$ & $2.69 \pm 0.39 \%$ & modern & & \\
\hline-10 & $160: 164$ & $1.48 \pm 0.50 \%$ & modern & & \\
\hline-11 & $160: 165$ & $2.66 \pm 0.44 \%$ & modern & & \\
\hline-12 & $160: 164$ & $3.96 \pm 0.45 \%$ & modern & & \\
\hline-13 & $160: 164$ & $0.96 \pm 0.39 \%$ & modern & & \\
\hline-14 & $160: 163$ & $30 \pm 57$ & & & A.D. 1920 \\
\hline-15 & $160: 164$ & $1.03 \pm 0.52 \%$ & modern & & \\
\hline-16 & $160: 164$ & see $\partial \mathbf{C}^{14}$ & & $+168 \pm 9$ & \\
\hline
\end{tabular}

